Journal of Theoretical and Applied Mechanics, Sofia, 2015, vol. 45, No. 3, pp. 37-52

\title{
INFLUENCE OF THIN-WALL STRUCTURE ON STRESS DISTRIBUTION OF CURVIC COUPLINGS*
}

\author{
SHUXIA YUAN \\ School of Mechanical Engineering, Xi'an Shiyou University, \\ Xi'an, PR China, \\ e-mail: flowingcloud269@163.com \\ Youyun Zhang, Yongsheng Zhu \\ Theory of Lubrication \& Bearing Institute, Xi'an Jiaotong University, \\ Xi'an, PR China, \\ e-mails: yyzhang1@mail.xjtu.edu.cn, yszhu@mail.xjtu.edu.cn
}

[Received 06 October, 2014. Accepted 15 June, 2015]

\begin{abstract}
The objective of this paper is to investigate the influence of transition structure between curvic teeth and disk on stress distribution of curvic couplings, and provide data to help improving the design of curvic couplings. In this work, the three-dimensional finite element method was used, and Augmented Lagrange Algorithm was adopted to the contact algorithm during the analysis. According to the results of this paper, designing thin-wall structure reasonably can avoid detaching of contact interface at external diameter during preload process; reduce stress fluctuation of curvic teeth caused by circumferential bolts structure; and balance stress difference of each contact pair caused by different disk quality under the action of the centrifugal force.

KeY WORDS: Curvic coupling, circumferential bolt, thin-wall structure, stress distribution, finite element method.
\end{abstract}

\section{Introduction}

The ultra-high temperature service units such as aero-engines and gas turbines need an ultra-precise locating arrangement to assure the exact alignment of components along the main engine shaft, whereas the misalignment will produce extra stress, which is enlarged during the condition of high temperature. In addition, the rotor is easy to achieve precise positioning in assembly,

\footnotetext{
${ }^{*}$ Corresponding author e-mail: flowingcloud269@163.com

This work was supported by the National Basis Research Program of China under the grant number of 2007CB707706. The authors are grateful for this support.
} 

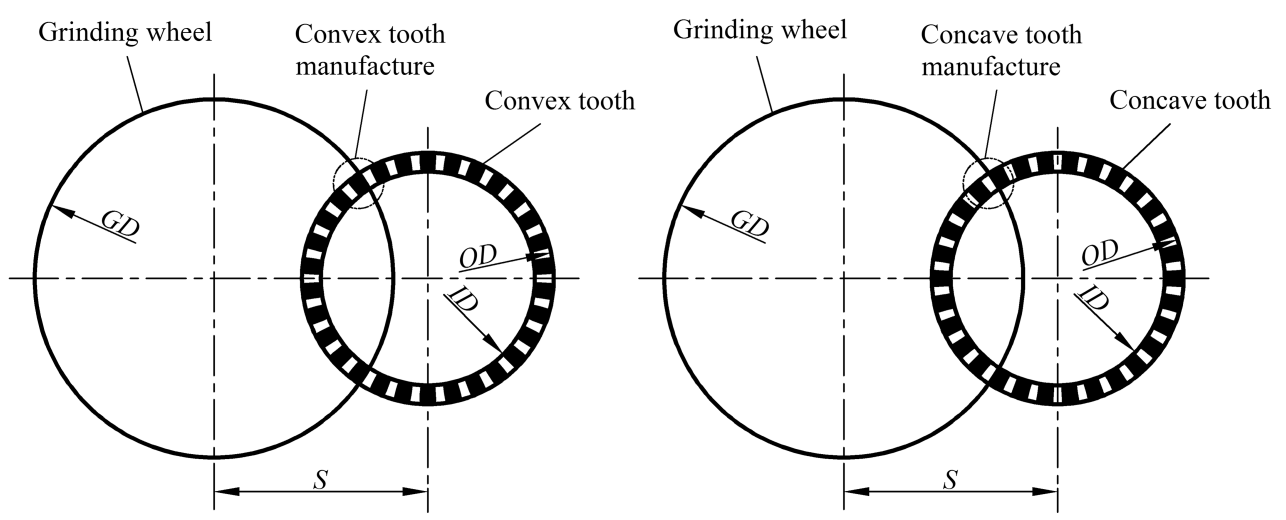

Fig. 1. Convex and concave tooth manufacture

and the alignment could be easily re-established during maintenance, to ensure continuous contact of each component, the solution was found in the curvic coupling. In these couplings, disks with curvic teeth are tied together by one central bolt, or several bolts, arranged circumferentially on the pitch circle through the disc webs. The matching teeth are configured with convex teeth on one-half and concave teeth on the other half, guarantees proper alignments, when the faces of two halves on the couplings are pressed together (Fig. 1). Furthermore, as the matching convex and concave teeth, the distance from coupling center line to outside diameter of the curvic teeth will always be the same, so to achieve the automatic alignment.

For this type of connection, failures may occur on curvic couplings as galled, fritted, and broken teeth [1]. The former two failures reduce the transfer accuracy and realignment abilities, whereas curvic broken teeth threaten safety of the unit directly. The failures of curvic couplings lie on its stress distribution, to study the stress distribution of curvic couplings, understand the reasons resulting the stress distribution is the premise of taking measures to avoid possible damaged stress generated. The design method of curvic couplings was published by Gleason Works in 1986 [2]. This is a general standard, and the stress distributions of curvic teeth needs to be studied further. However, for the very complicated structure of curvic couplings, it is difficult to achieve the complex three-dimensional stress distribution through analytic method, so very little improvement or detailed analysis of the stresses distribution of curvic couplings was published for a long time. It is possible to analyze curvic couplings using the finite element method with the development of compute capabilities and finite element analysis software in analyzing complex three-dimensional 
contact problems. And in reference [3], the authors compare the stress of curvic couplings obtained from FEM with photo-elastic experiment and validated the feasibility of computing contact stress of curvic couplings using FEM. Yuan, et al. [4] investigated the stress distribution of curvic teeth under various working conditions. Muju [5] disclose a curvic coupling with compound fillet radius, which is a lower stress and higher service life curvic coupling design. Jiang, et al. [6] analyzed the contact stresses of curvic couplings in a blade-off event. Kenneth, et al. [7] discussed the tooth engagement and load shearing of involute spline, the torque transmitting process of the involute spline is similar to curvic couplings, but it does not require preload. Jiang, et al. [8] investigated the stiffness of curvic couplings in tightening, and loosening mechanism of bolt in curvic coupling subjected to transverse loading [9].

In order to ensure the coupling accuracy of aero-engines and gas turbines, curvic teeth and disk are usually formed integrally. For proper alignments, radial dimension of curvic teeth is small (for example, the rotor studied in this work with outer diameter of $1200 \mathrm{~mm}$ and inner diameter of $1140 \mathrm{~mm}$ ), whereas radial dimension of disks is large (with outer diameters about of 2000 $\mathrm{mm}$ and inner diameter of $144 \mathrm{~mm}$ ) to bear centrifugal load caused by disks and blades. Curvic couplings and disks are showed in Fig. 2. There are some differences on stiffness of the curvic teeth with small radial size and the disks with large radial size. Transitions between the two parts also affect the stress distribution of curvic coupling and the bearing abilities of the rotor. Furthermore, the service life of the coupling is influenced. There are several kinds of transitions that can be used between the two parts. The simplest structure is a direct connection of the two parts, which is shown in Fig. 3 (a). In addition, a

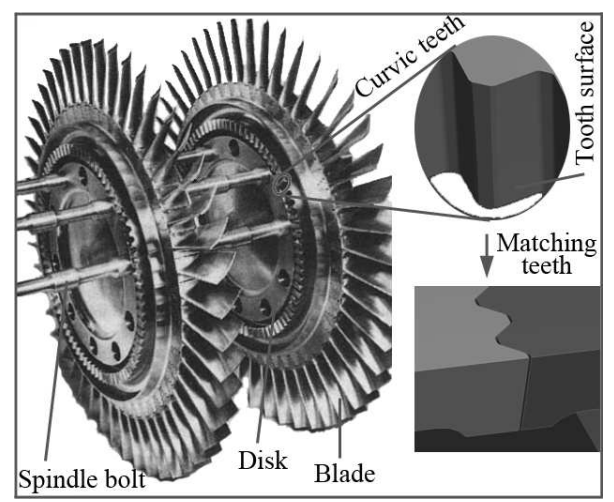

Fig. 2. Curvic couplings in rotor 


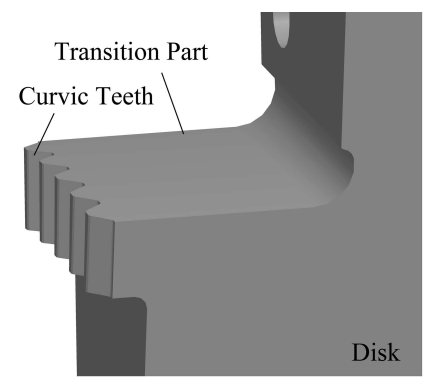

(a)

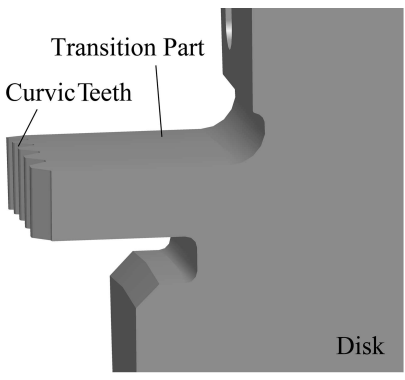

(b)

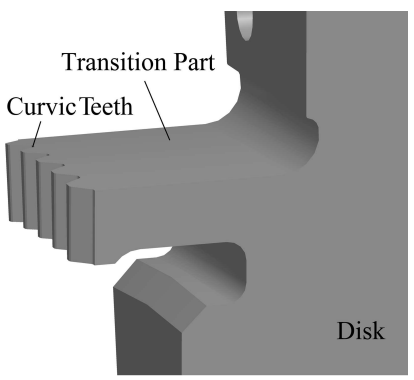

(c)

Fig. 3. Various transitions between curvic teeth and disk, (a) Direct connection, (b) Equal thickness transition structure, (c) Thin-wall transition structure

certain length of transition part with the same thickness as curvic teeth can be set between the two parts, and this structure is illustrated in Fig. 3 (b). On this basis, the transition part can be thinned to a thickness less than the thickness of curvic teeth, and this structure is called "thin-wall structure" in this paper, which is shown in Fig. 3 (c). For the different stiffness of each structure, different transition structure will result the different stress distribution of curvic couplings. Besides, some structures with stiffness between Figs 3 (a) and 3 (b) may be adopted as the transitions, but according to the researching result, these structures are even inferior to the structure of Fig. 3 (b). So the three representative transitions were discussed. Detailed geometrical parameters are presented in Fig. 4. $D$ and $d$ represent the outer and inner diameters of curvic teeth ring, $T$ is the depth of the curvic teeth. The thickness of the thin-wall is $\delta$, which is less than $(D-d) / 2$, and it can be adjusted according to the stress distribution of curvic teeth. The length of the thin-wall, i. e. $L$, is determined by different mass of the disk connected by it.

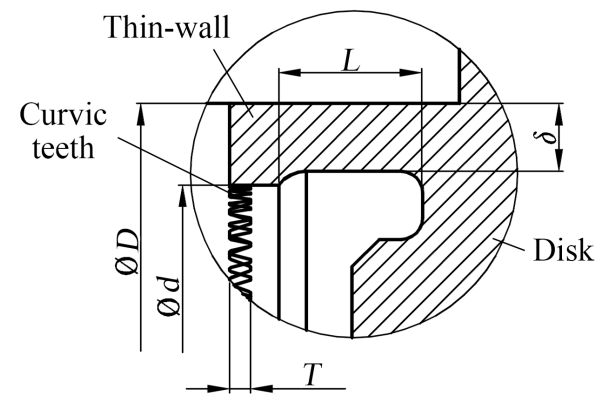

Fig. 4. Geometrical parameters of thin-wall transition structures 
Influence of Thin-Wall Structure on Stress Distribution of Curvic Couplings 41

In this paper, the stress change tendency of curvic couplings with different transition structure during the preload stage and the work process is studied through three-dimensional finite element analysis. Moreover, technics such as optimizing the mesh, setting contact parameters reasonably, was taken to make the calculation converged. The research work of this paper could give a better understanding of stress distribution, caused by transition structure and provide some references for the design of curvic couplings.

Transition structure can affect stress distribution of one curvic tooth, stress distribution between curvic teeth, and stress comparison of different contact pairs. The influence of different structure on stress distribution was discussed and evaluated.

\section{Finite element analysis}

In this analysis, a geometrically complex three-dimensional finite element model of curvic couplings rotor was constructed through ANSYS commercial software, this is a gas turbine rotor, and teeth number of the curvic couplings is 180 , the rotor is assembled and preloaded by twelve spindle bolts, and its cutaway view is illustrated in Fig. 5 (a). Some complex features, disinterested in this study were simplified in modelling based on Saint-Venant principle, but curvic teeth and thin-wall structures are the researching key point, which were not simplified. The FEM model is illustrated in Fig. 5 (b) and upper right corner in Fig. 5 (b) is sectional enlargement meshing figure of one curvic tooth. In reference [4], the author compared the mesh quantity of curvic teeth with the work of reference [3] and validated that the mesh of curvic teeth showed in Fig. 5 (b) can meet the requirement precision of FEM.

The contact definition was applied between the teeth of the two coupling halves, between the spindle bolts and sidewall and between the bolt press surface and nuts of the spindle bolt. Augmented Lagrange Algorithm was selected as the contact algorithm during the analysis, for it is explained in the ANSYS Help document that the Augmented Lagrange Method usually leads to better conditioning and is less sensitive to the magnitude of the contact stiffness compared to the Penalty Method. At these contact pairs, the friction coefficient is set to 0.2 ; normal penalty stiffness factor is 1 , and penetration tolerance factor is 0.1 . For proper constraining the rotor, the compressor end without curvic teeth was also included in this model. In this analysis, all degree of freedom except axial direction on the two bearings in Fig. 5 were constrained, and axial degree freedom on the left end of the rotor was constrained. The loads applied to the finite element model were bolt clamping force and centrifugal load. The 


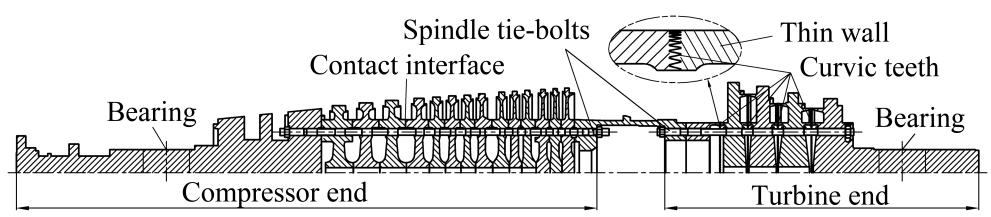

(a)

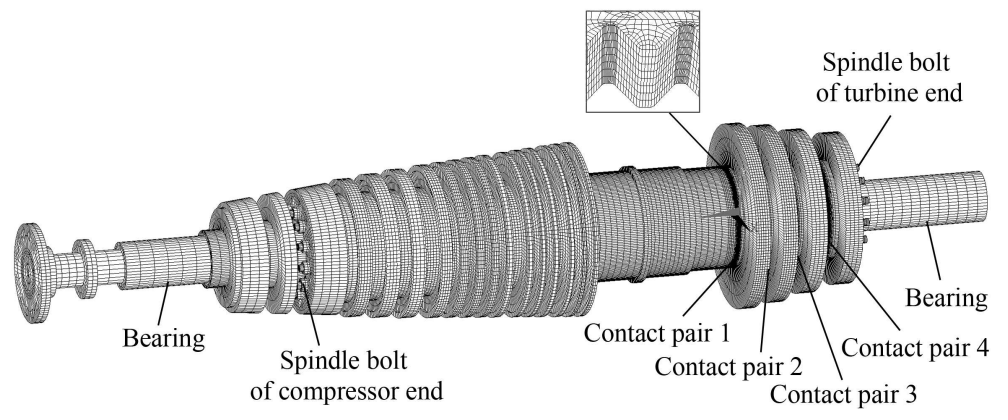

(b)

Fig. 5. A model of heavy-duty gas turbine rotor (a) Cutaway view of the rotor, (b) Modelling and meshing of the rotor

centrifugal load was simulated by the applied angular acceleration. The bolts clamping force were applied by inserting PRETS 179 element of ANSYS software into the spindle bolts of the finite element model, which can apply the preload force accurately. For circumferential bolt connecting structures, the bolt number is greater than 3 , and if the bolts are not preloaded simultaneously, the bolt's clamping force preloaded previously would be changed with the preloading processes subsequently, this phenomenon is called elastic interaction. Elastic interaction will affect the stress distribution of the rotor and curvic teeth. Reference [10] has discussed the influence of elastic interaction on the stress distribution of curvic teeth, bolts and rotors, and proposed a method to achieve uniform clamp force. The bolt was applied as uniform clamping force, because this paper is the only concern of the effect of thin-wall. To avoid disturbed by extra factors, the load step is set as automatic time stepping, number of time stepping is 5 , and this number will be changed with the convergence process.

Analyses have been carried out to show the stress distribution of the curvic couplings under different load conditions and effect of geometrical changes of transition part to the stress distribution of curvic couplings. In this paper, the preload process and speed-up process was analyzed to study the influence of thin-wall structure on stress distribution of curvic couplings. In general, the 
Influence of Thin-Wall Structure on Stress Distribution of Curvic Couplings 43

Table 1. Material properties used for the analysis

\begin{tabular}{|c|c|c|c|}
\hline Items & \multicolumn{2}{|c|}{ Bolt material } & Disk material \\
\hline Elasticity modulus $E(\mathrm{GPa})$ & \multicolumn{2}{|l|}{204} & 198 \\
\hline Poisson's ratio $\nu$ & \multicolumn{2}{|l|}{0.3} & 0.375 \\
\hline Density $\gamma\left(\mathrm{kgm}^{-3}\right)$ & \multicolumn{2}{|l|}{8240} & 7930 \\
\hline$\sigma_{0.2}(\mathrm{MPa})$ & \multicolumn{2}{|l|}{1050} & 950 \\
\hline \multirow{4}{*}{ Elastic-plastic data } & Stress (MPa) & Strain & \\
\hline & 1050 & 0.0058 & \\
\hline & 1090 & 0.0060 & \\
\hline & 1110 & 0.0062 & \\
\hline
\end{tabular}

curvic couplings work at thermal conditions, however, from the previous work of Yuan [4] it can be known that the stress variation of curvic couplings between the two applied forces, i.e. preload force and centrifugal force, under normal and thermal condition is almost equal. Therefore, the temperature load does not affect the stress change tendency of the rotor. Thus, the thermal condition was not involved in this work.

Material properties of the heavy-duty gas turbine rotor are listed in Table 1. For stress of spindle bolt is usually greater than disk's stress, material strength of spindle bolt is higher than that of disks. According to the results of finite element analysis, there is some plastic deformation on the root of the spindle bolts' nut, so part of the stress-strain values of the spindle bolt material are also listed in Table 1.

The bolts are supposed to supply enough clamping force to ensure sufficient contact force between the matching curvic teeth, meanwhile, the von Mises stress of the bolts and the connecting parts should not exceed the allowable stress of the materials. According to the research work of Yuan et. al. [4], operating temperature of curvic teeth and tie-bolts is about $300^{\circ} \mathrm{C}$, and yield strength of tie-bolts and disks at this temperature is $970 \mathrm{MPa}$ and $915 \mathrm{MPa}$, respectively. Taking some unsteady operating conditions and fault conditions into account, safe coefficient was taken as 1.5, and then the allowable stress of tie-bolts and disks is $650 \mathrm{MPa}$ and $610 \mathrm{MPa}$, respectively. In this work, the preload is determined by the stress status of the curvic teeth, and von Mises stress in the hoop direction is expressed in Fig. 6. The maximal preload force is achieved by increasing the preload force gradually, if von Mises stress on any part of the tooth approach to the allowable stress of the material. The diameter of the bolt can be designed according to the obtained force and the allowable stress of the bolt material. When comparing the stress between curvic teeth, the average stress of every point on the contact surface is used, so the value is 


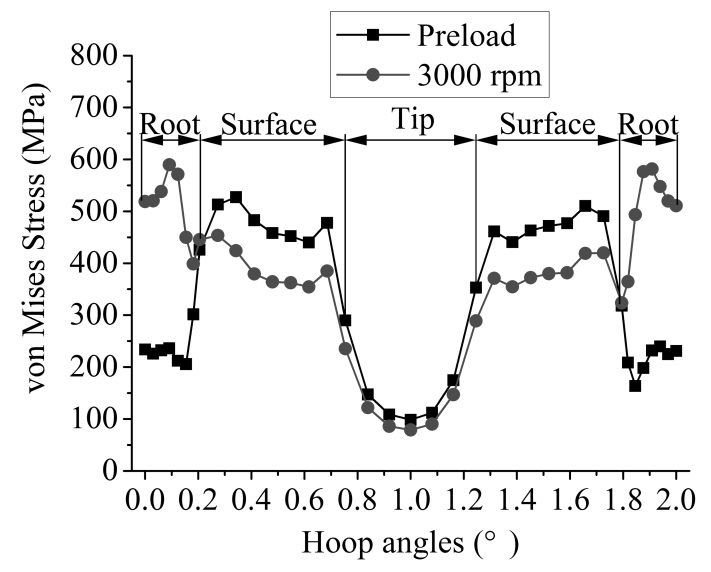

Fig. 6. Stress distribution of a tooth along the hoop direction

not too large, in fact, the local stress is close to the allowable stress.

\section{Results and analysis}

\subsection{Influence of thin wall structure on radial stress distribu- tion of curvic tooth}

For a bolted rotor with curvic couplings, the curvic teeth and spindle bolts may lay on different position in the radial direction, and in generally, the spindle bolts are located inside the ring formed by curvic teeth (Fig. 5). Thus, the preloads are applied off to one side of the curvic teeth, which are called 'prying loads' [11], due to bending effect, the stress variation from internal diameter to external diameter of the curvic teeth ring is formed. The transition structure between curvic teeth and disk also affects stress distribution of curvic teeth at radial direction. Relations between different transition structure and radial stress distribution of curvic couplings are shown in Fig. 7. Figure 7 (a) indicates the nodes' position on curvic tooth, and Fig. 7 (b) is von Mises stress of the nine nodes in Fig. 7 (a). It can be noticed that stress of curvic tooth decrease sharply from internal to external diameter (Trans-1 in Fig. 7 (b)) without thin-wall part (this structure can be seen in Fig. 3 (a)), this is because the stiffness change abruptly from disks to curvic teeth. Because the spindle bolts are locating at internal side of curvic teeth ring, the curvic couplings and spindle bolts are not working on the same circle during preload process, and thus a bending moment will be produced. Therefore, contact stress of internal diameter is greater than that of external diameter, which may lead to the departure of contact surface at external diameter position in some cases 


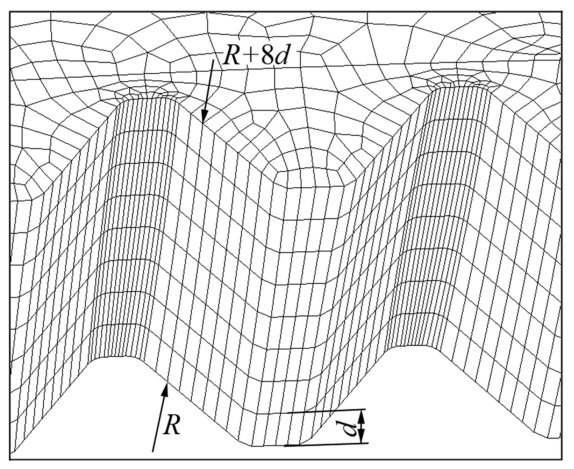

(a)

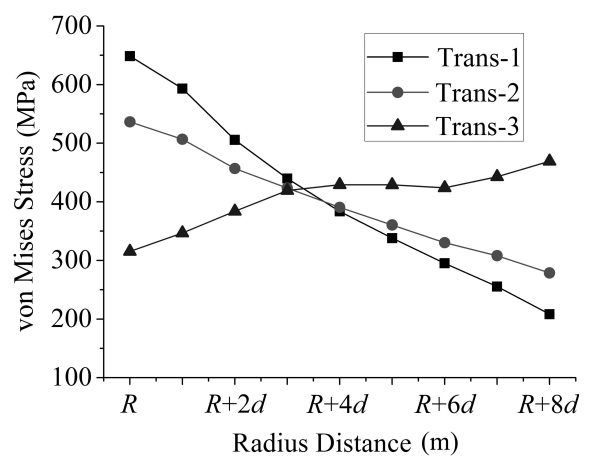

(b)

Fig. 7. Von Mises stress comparison of curvic tooth at radial direction for various transition structures

and affect bearing abilities of curvic couplings seriously. When adopting thinwall structure between curvic couplings and disks, and thickness of thin-wall is equal to that of curvic teeth (Fig. 3 (b)), stress variation at radial direction is moderated for the deformation in thin-wall section during preload process, but stress of curvic teeth in internal diameter is still greater than that of external diameter (Trans-2 in Fig. 7 (b)). If thickness of thin-wall is thinner than that of curvic teeth in internal diameter position (Fig. 3 (c)), stress distribution of curvic couplings at radial direction is changed completely (Trans-3 in Fig. 7 (b)). It does not only reduce the stress variation at radial direction but also change the maximum stress from internal to external diameter position for the reducing stiffness of internal diameter improving the deformation capacity of the thin-wall part, and avoid that stress of external diameter is too small to detach during preload process.

\subsection{Influence of thin-wall structure on stress distribution be- tween curvic teeth}

The circumferentially bolts' structure change the stress distribution between curvic teeth, which is to say that there exist stress differences between each tooth. In addition, this stress difference is changed with the geometrical changes of the transition part. Therefore, this paper studied stress distribution between curvic teeth caused by the different transition structure; interpret the influence of transition structures on stress distribution of curvic couplings further.

Each contact pair of the rotor, studied in this paper is comprised of convex and concave teeth and each part contains 180 teeth, respectively. The 


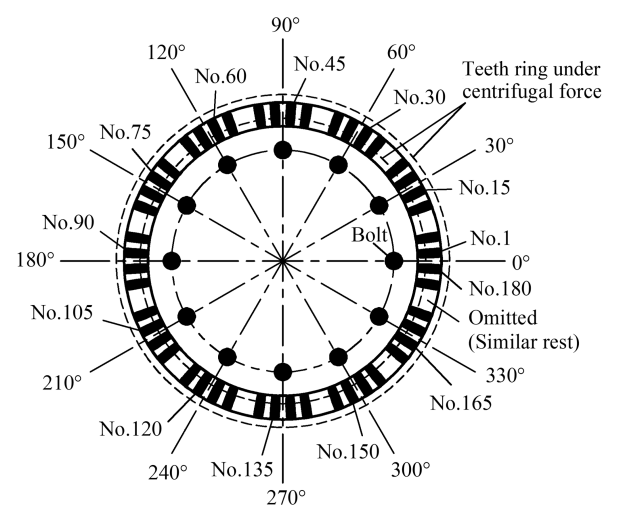

Fig. 8. Numbering and relative position of curvic teeth

180 teeth were numbered from zero degree as No. 1 to No. 180 and shown in Fig. 8, and relative position of each tooth with bolts is illustrated in Fig. 8, too.

According to the result of this research, stress change tendency of each contact pair is similarity, although some differences in value exist. Take contact pair 2 as example, other contact pairs are similar to the example, and differences between each contact pair were studied also. Hereinafter, $X$ is radial direction, $Y$ is hoop direction, and $Z$ is axial direction. Direct stress of each direction in cylindrical coordinates system is expressed as $S_{X X}, S_{Y Y}$, and $S_{Z Z}$, respectively, and shear stress is expressed as $S_{X Y}, S_{Y Z}$ and $S_{Z X}$, respectively. Von Mises stress of the 180 teeth under preload force and centrifugal force is shown in Fig. 9, and stress value in Fig. 9 is average von Mises stress of nodes on contact surface (for clarity of the drawing, identifiers in Fig. 9 were set every five teeth, other point is represented by broken lines, similarly hereinafter).

It can be seen in Fig. 9 that the stress is almost equal at every tooth and is not affected by the preload force of 12 dispersed spindle bolts after preloading. Whereas influence of centrifugal force on stress of curvic teeth is attributed to two aspects, the first is the decreasing of stress on the contact surface, and the second is that it formed obviously stress wave, for it is expressing evident stress peaks and valleys on the 180 teeth. Together with Fig. 8, it can be seen that von Mises stress of tooth locating at bolts position is smaller. Reasons of the two phenomena can be explained as follows: the disks are elongated at radial direction under the effect of centrifugal force and shorten in axial direction for Poisson effect, thus, the direct stress $S_{Z Z}$ is decreased. For the elongation of disks at radial direction, radial position of curvic teeth ring changed from real to 


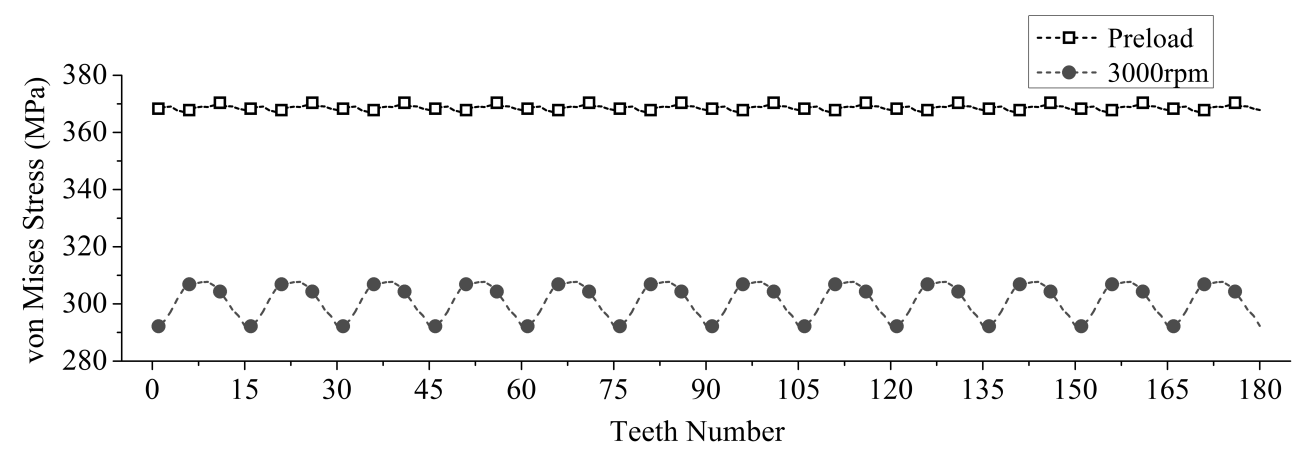

Fig. 9. Von Mises stress of the curvic teeth, under the effect of preload and centrifugal force

broken line in Fig. 8. Increasing of radial diameter leads to the decrease of hoop compression force, so does the hoop stress $\left(S_{Y Y}\right)$. Furthermore, for the existing of spindle bolts, mass in hoop direction is uneven and appeared cycle trend. Total mass of the rotor at bolt position is greater than middle position, because material density of spindle bolts is greater than that of disks and axial length of spindle bolts is longer than sum of all stages disks' length. During the process of speed-up, centrifugal force of the spindle bolts act on disks, which increase the radial dimension of disks at bolts' positions, i. e. more radial deformation, so the decreasing of $S_{Y Y}$ and $S_{Z Z}$ in bolt position is also greater than that in middle position for the reason explained formerly, and forms stress fluctuation as shown in Fig. 9. The fluctuation exists in the whole operating process and is inevitably in this kind of structure, but it can be diminished by adopting material density of spindle bolts equal to, or less than that of disks or increasing number of spindle bolts properly during design process. Stress distribution of curvic couplings with doubled number of spindle bolts is illustrated in Fig. 10, it can be seen that stress fluctuation is reduced in a certain extent.

In addition, thin-wall structure between curvic teeth and disks also can reduce the stress fluctuation caused by circumferential bolts. For the three transition structures mentioned above, stress distribution between each curvic tooth of them was studied, and the result is shown in Fig. 11. As can be seen, without thin wall part, the curvic teeth present maximum von Mises stress and maximum stress fluctuation (Trans-1 in Fig. 11). With equal thickness transition structure (i.e. the Trans-2 in Fig. 11), the von Mises stress, and stress fluctuation of curvic teeth are all reduced in some extent. While for the thinwall structures (Trans-3 in Fig. 11), the von Mises stress, and stress fluctuation of curvic teeth are reduced further, so this stress distribution is the best among 


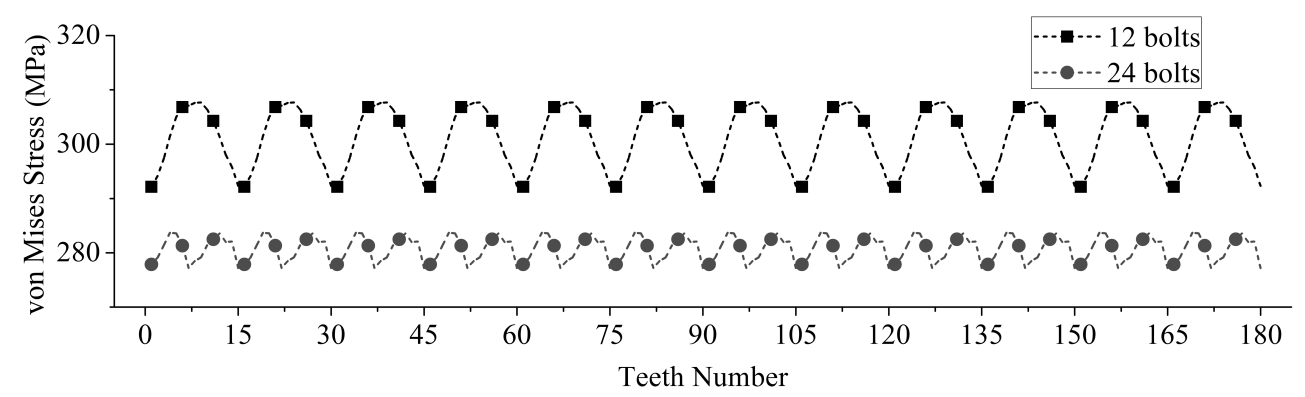

Fig. 10. Von Mises stress comparison of curvic teeth with 12 or 24 bolts under the rotating speed of $3000 \mathrm{rpm}$

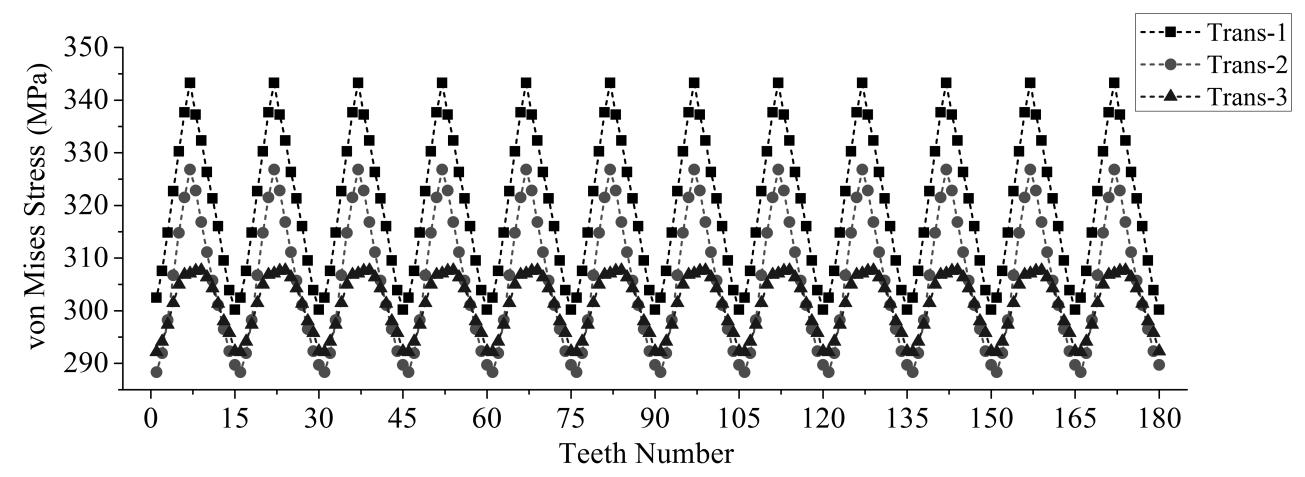

Fig. 11. Von Mises stress comparison of curvic teeth with different transition structure, under the rotating speed of $3000 \mathrm{rpm}$

the three transition structures. Reason to generate these stress distribution is due to the thin-wall structure would produce a bending deformation, which makes the length of the transition section shorten, and also reduces the axial force acted on the curvic couplings, thus, the von Mises stress of curvic couplings is reduced accordingly. In addition, the thin-wall structure with greater flexibility, acting as a buffer between the large stiffness disk and curvic teeth with small stiffness, prevents portion of the stress fluctuation transmit from the disk to the curvic teeth. Therefore, the thin-wall structure can also effectively reduce the stress fluctuation of curvic couplings caused by circumferential bolts, under the effect of centrifugal force. 
Influence of Thin-Wall Structure on Stress Distribution of Curvic Couplings 49

\subsection{Influence of thin-wall length on stress distribution of cur- vic couplings}

The lengths of thin-wall structure of each contact pair are supposed to be different, to study the influence of thin-wall length on stress distribution of curvic couplings. Thin-wall structures, settings on both sides of the contact pairs 1 and 4, are longer than that of contact pairs 2 and 3 . It is indicated in Fig. 12 (a) that von Mises stress of contact pairs 2 and 3 is greater than that of contact pairs 1 and 4 after preloading. Stress contrasting of each contact pair after preloaded is illustrated in Fig. 13, the results show that the stress difference is caused by the different hoop stress $\left(S_{Y Y}\right)$. For the thin wall of contact pairs 1 and 4 is longer than that of contact pairs 2 and 3 , and the long thin wall is easily formed as the bending like drum shape (Fig. 14) during preload stage, the longer thin wall, the more drum bending. Because of the drum bending, the radius of curvic couplings contact pairs 1 and 4 are increased, so the hoop stress of them are decreased for the decreasing of hoop compression force between curvic teeth. Axial stress is the same in contact pairs 1 to 4 for the same preload force, when hoop stress is decreased, von Mises stress is decreased, accordingly. Thus, it can be known that lower stress of contact pairs 1 and 4 is induced by the drum-bending phenomenon during preload.

From the deformation characteristic of curvic couplings' thin wall during preload process, some inspires could get that the stress in contact surface of each contact pair during preload process can be controlled through changing the length of thin wall, and the merit is manifested in turbo machinery. For example, in the heavy-duty gas turbines, the blade length is usually different in each stage, in order to ensure the casing diameter not to change too much, the disk diameter of each stage is usually not the same, the shorter blade length, the larger disk diameter, and the longer blade length, the smaller disk diameter. Due to the different disk size of each stage, which leads to the different mass distribution, radial deformation of each disk is different under centrifugal force, so does the stress decrease of each contact pair. Therefore, the adjusting function of the thin-wall can be used by the designer to adjust the length of thin-wall of each contact pair, according to the mass distribution of each disk, the larger mass of disk, the shorter thin wall. Thus, stress of curvic couplings at contact pairs 2 to 3 is larger during preload process, and stress decrease much under centrifugal force for the large mass of that disk, so a uniform stress distribution of each contact pair is obtained at last. It is shown in Fig. 12 (b) that the design of this structure achieves its intention (there is disk only on one side of contact pair 1, so stress fluctuate of contact pair 1 is smaller). 


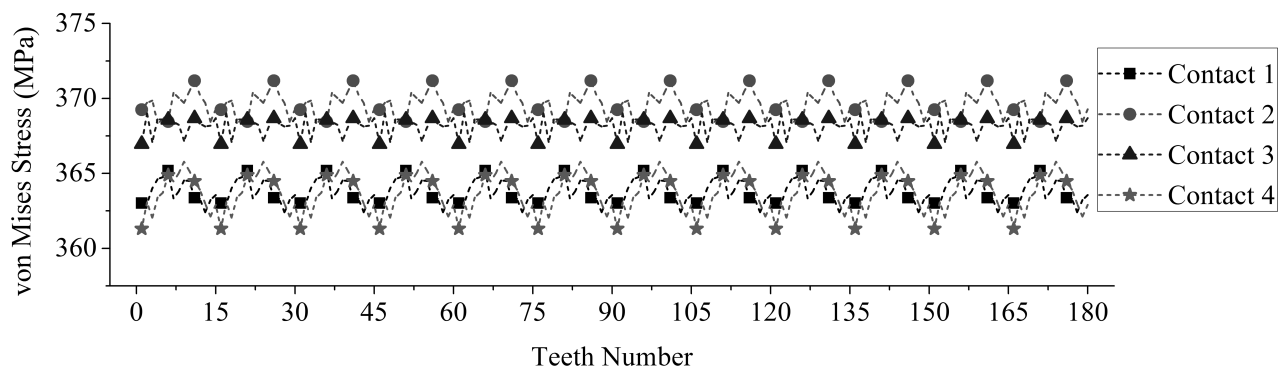

(a)

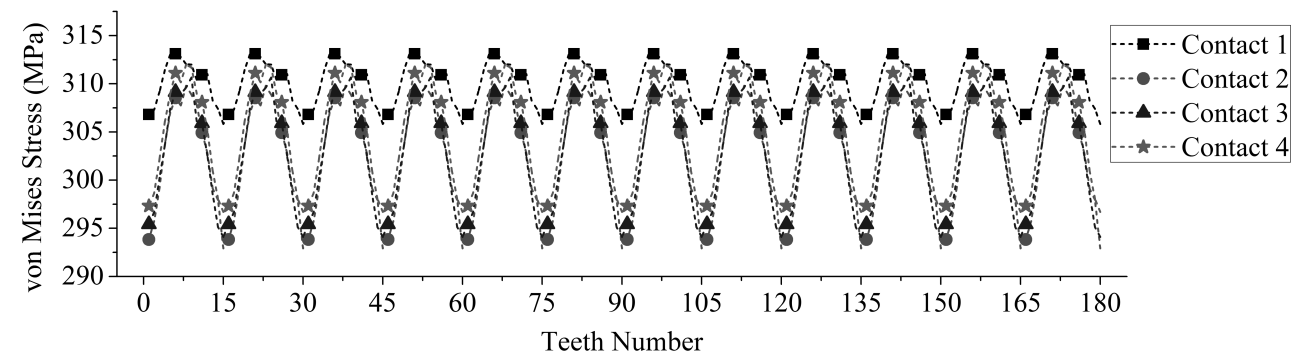

(b)

Fig. 12. Von Mises stress comparison of curvic teeth in contact pairs 1-4 (a) Preloaded, (b) Speed-up to $3000 \mathrm{rpm}$

\section{Conclusion}

The influence of thin-wall structure on stress distribution of curvic couplings was analyzed through three-dimensional finite-element method, by modelling the curvic couplings rotors with different types of transition structures, applying contact definition between contact interfaces, and simulating the conditions of preload and work process. It can be obtained from the result that thin-wall structure can adjust radial stress distribution of curvic teeth, reduce stress fluctuation of curvic teeth caused by circumferential bolts structure, under the action of centrifugal force, and balance the stress difference of each contact pair caused by different disk quality, under the action of the centrifugal force. The results of this paper may provide the following references for the design of curvic couplings:

(1) To get a reasonable radial stress distribution of curvic teeth, the thin-wall structure could be designed between the curvic teeth and the disk. For the most commonly structure, the spindle bolts are located inside the ring formed by curvic teeth, the thin-wall structure will not only reduce the stress variation at radial direction but also will change the maximum stress from 
Influence of Thin-Wall Structure on Stress Distribution of Curvic Couplings 51

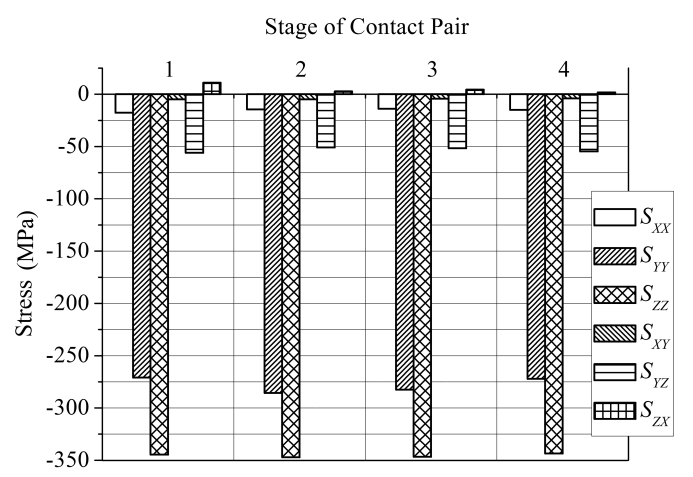

Fig. 13. Stress contrasting of each contact pair after preloading

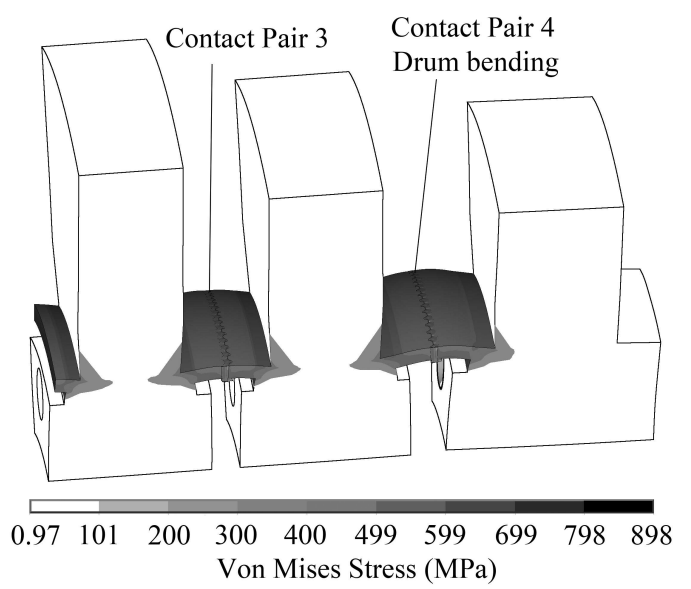

Fig. 14. Obvious drum bending of contact pairs 3 and 4 after preloading

internal to external diameter position, and avoid that the stress of external diameter is too small to detach during preload process.

(2) Thin-wall transition structure between curvic teeth and disk can block part of the stress fluctuation transmit to curvic teeth, therefore the stress fluctuation of curvic couplings, caused by circumferential bolts under the effect of centrifugal force, is reduced through adopting this structure.

(3) In a bolted rotor with curvic couplings, when disks have unequal diameters, different length of thin-wall is configured on each curvic teeth contact pair, which can balance the stress difference of each contact pair caused by different disk quality, under the action of the centrifugal force, and ultimately a uniform stress distribution of each contact pair is obtained. 


\section{REFERENCES}

[1] Aircraft Incident/Accident Report: NTSB Narrative Summary Released at Completion of Accident, 2007, http://www.aircraftone.com/aircraft/ accidents/20070823X01225. asp.

[2] Works, G. Curvic Coupling Design, New York, Rochester, Gear Technology, Gleason Works, 1986, 34-48.

[3] Richardson, I. J., T. H. Hyde, A. A. Becker, J. W. Taylor. A Validation of the Three-dimensional Finite Element Contact Method for use with Curvic Couplings, Proceedings of the Institution of Mechanical Engineers Part G-Journal of Aerospace Engineering, 216(G2), 2002, 63-75.

[4] Yuan, S. X., Y. Y. Zhang, Y. C. Zhang, X. J. Jiang. Stress Distribution and Contact Status Analysis of a Bolted Rotor with Curvic Couplings, Proceedings of the Institution of Mechanical Engineers Part C-Journal of Mechanical Engineering Science, 224(C9), 2010, 1815-1829.

[5] Muju, S., R. S. Sandoval. Curvic Coupling Fatigue Life Enhancement through Unique Compound Root Fillet Design, Patant Application, H. I. Inc., ed. U. S, 2003, p. 9.

[6] Jiang, X. J., Y. Y. Zhang, S. X. Yuan. Analysis of the Contact Stresses in Curvic Couplings of Gas Turbine in a Blade-off Event. Strength of Materials, 44 (2012), No. 5, 539-550.

[7] Chase, K. W., C. D. Sorensen, B. DeCaires. Analysis of Tooth Engagement and Load Sharing in Involute Splines, Gear Technology, Randall Publications LLC, IL 60007 USA, 1840 Jarvis Avenue Elk Grove Village, 2010, 54-62.

[8] Jiang, X., Y. Zhu, J. Hong, Y. Zhang. Development and Validation of an Analytical Model for Stiffness Analysis of Curvic Coupling in Tightening. Journal of Aerospace Engineering, 27 (2014), No. 4, (04014012) 01-14.

[9] Jiang, X., Y. Zhu, J. Hong, X. Chen, Y. Zhang. Investigation into the Loosening Mechanism of Bolt in Curvic Coupling Subjected to Transverse Loading. Engineering Failure Analysis, 32 (2013), 360-373.

[10] Yuan, S. X., Y. Y. Zhang, Y. G. Fan, Y. C. Zhang. A Method to Achieve Uniform Clamp Force in a Bolted Rotor with Curvic Couplings, Proceedings of the Institution of Mechanical Engineers Part E - Journal of Process Mechanical Engineering, Online First, 2014, DOI: 10.1177/0954408914550017 uk.sagepub.com/jpme.

[11] Croccolo, D., M. De Agostinis, N. Vincenzi. A Contribution to the Selection and Calculation of Screws in High Duty Bolted Joints. Int. J. Pressure Vessels Pip., 96-97 (2012), 38-48. 